Адрес статьи / To link this article: http://cat.itmo.ru/ru/2021/v6-i2/273

\title{
Эффективность использования технологий виртуальной реальности в методиках лечения дентофобии для облегчения психофизиологического состояния пациентов стоматолога
}

\author{
Н.А. Туликов, А.А. Смолин \\ Университет ИТМО, Россия \\ mantis373@gmail.com, smolin@itmo.ru
}

\begin{abstract}
Аннотация. Виртуальная реальность - актуальное направление в сфере современных компьютерных технологий, которое широко используется во многих сферах, особенно в медицине. Целью данной работы является проверка и оценка эффективности использования технологий виртуальной реальности в методиках лечения дентофобии путём погружения пациента, страдающего дентофобией, в виртуальную среду, имитирующую операционный кабинет врача-стоматолога. Вследствие того, что многие пациенты испытывают страх перед операциями, было принято решение использовать технологии виртуальной реальности в предоперационной подготовке. В процессе работы было разработано приложение виртуальной реальности для гарнитуры Samsung Gear VR, изучены способы применения виртуальной реальности и методы взаимодействия с ней, психологические терапевтические теории, проведён анализ ПО для разработки приложения.
\end{abstract}

Ключевые слова: виртуальная реальность, дентофобия, терапия, медицина, виртуальная реальности, фобия, стоматолог, предоперационная подготовка, пациенты, междисциплинарные исследования.

\section{Введение}

Одной из базовых, фундаментальных эмоций, сопровождающей человека всю его жизнь является страх. Страх - это внутреннее состояние индивида, индивидуальная эмоция, обусловленная реальным, или предполагаемым грозящим ему бедствием или опасностью, возникающая при наступлении, или просто при предвкушении трудноразрешимых ситуаций. С точки зрения современной психологии он, однако, не всегда считается отрицательно окрашенным эмоциональным процессом, хотя преобладающее большинство людей наделяют испытываемое тревожное состояние негативной окраской. Страх в животном мире - это эмоция, которая основывается на прошлом негативном опыте. Она играет очень важную роль в самом выживании особи.

Частным случаем интенсивного, панического страха, возникающего и усиливающегося при приближении или наступлении конкретных определённых событий и не поддающегося контролю, пониманию и логическому объяснению, является фобия. Человек может испытывать 
Эффективность использования технологий виртуальной реальности в методиках лечения дентофобии для облегчения психофизиологического состояния пациентов стоматолога

тревогу, даже панический страх при контакте с, казалось бы, простыми и совсем не опасными вещами или в простых повседневных ситуациях.

Примером такой фобии является дентофобия - боязнь стоматологического лечения. Примерно 75\% людей испытывают дискомфорт при посещении стоматолога, 20 процентов людей страдают от дентофобии [7]. Она может возникнуть у людей в связи с неприятным опытом, особенно в детстве, когда человек особенно чувствителен и восприимчив. Первые симптомы тревожных расстройств часто проявляются после существенных психических переживаний, длительного стресса, перемен в образе жизни, например, после переезда в другой город. Также свою роль играют родители, которые могут сами бояться стоматолога, чей страх передаётся ребёнку. Или же просто нежелание человека демонстрировать врачу плохое, запущенное состояние своих зубов. Нетактичный врач может спровоцировать или усилить ситуацию, которая может стать пусковым механизмом для возникновения фобии.

Человек, страдающий дентофобией, оказавшись в стоматологическом кресле, а порой, просто в кабинете стоматолога, не расслабляется, а наоборот, напрягается, не может установить необходимый контакт с врачом, не позволяет выполнить необходимые процедуры. От абсолютно любых, даже самых безопасных и безболезненных действий стоматолога, у такого нетипичного пациента проявляются интенсивные соматические проявления, вплоть до потери сознания. Дентофобу достаточно бывает просто увидеть инструменты или оборудование в кабинете, услышать звук бурмашины, как он теряет над собой контроль, вплоть до применения физической силы.

Пытаться справиться с дентофобией путём отказа от необходимых для здоровья осмотров стоматолога не удастся, так как заботиться о своём здоровье должен каждый человек. Поэтому пациенту, в случае боязни стоматолога необходимо бороться со своим психическим расстройством, тем более что сейчас существуют методы лечения, которые позволяют полностью устранить проявления страха.

\section{Использование технологии виртуальной реальности в медицине}

Виртуальная реальность - актуальное направление в сфере современных компьютерных технологий, которое очень широко используется во многих сферах, особенно в медицине и образовании. Социально значимой и наиболее развитой на данный момент областью применения систем виртуальной реальности в психологии и медицине является психотерапевтическая помощь при страхах, фобиях, посттравматических стрессовых расстройствах, хронических болях, борьбе с наркоманией и со стрессовыми заболеваниями. Стресс — это неспецифическое (аномальное) состояние или реакция организма на различные неблагоприятные факторы (стрессоры), воздействующие на него.

В связи с тем, что большинство вмешательств в челюстно-лицевой области сопровождаются болезненными ощущениями, нервно-психическое напряжение, тревогу и страх на приеме у стоматолога и челюстно-лицевого хирурга испытывают до 75\% пациентов, 20\% пациентов страдают от дентофобии. Во время лечения этих пациентов отмечаются изменения физиологических показателей, таких как: дыхание, частота сердечных сокращений, давление, повышенное потоотделение и другие вегетативные реакции, обострение уже имеющихся соматических патологий, а также формирование стойкого страха перед лечением заболеваний полости рта и других органов. Все это сказывается на работе врача, снижая качество оказываемых услуг. На данный момент существуют различные способы снижения уровня стресса в предоперационном периоде, такие как общение с медицинским психологом и профилактическая премедикация.

Помимо медикаментозной терапии сейчас все более распространенными становятся неинвазивные методы воздействия на уровень стресса, в том числе современные интерактивные технологии, в частности технологии виртуальной реальности.

Одним из актуальных направлений терапевтического использования виртуальной реальности является лечение фобий путем показа пациентам виртуальных изображений предметов, вызывающих у них непреодолимый страх (фобию). Лечение страхов с помощью

International Culture \& Technology Studies, Vol. 6, No. 2 
виртуальной реальности происходит через так называемый механизм привыкания (систематической десенсибилизации) или схему оперантного научения, которая предполагает постепенное привыкание человека к предметам и ситуациям, вызывающим у него страх на основе подкрепления реакций. Технической основой виртуальной реальности являются технологии компьютерного моделирования и компьютерной симуляции, которые позволяют реалистично отображать движение на экране. Виртуальная модель имитирует среду, в которой будет находиться пациент во время операции. Когда пациент привыкает к окружающей среде, он перестаёт воспринимать её как опасную для себя и страх исчезает.

В созданном VR пространстве пациент осознаёт, что виртуальный мир - это не настоящая реальность, он находится в подконтрольной среде, где ему на самом деле нечего боятся, что позволяет ему гораздо легче контролировать своё эмоциональное состояние и реакции. Вдобавок к этому, виртуальная среда полностью подконтрольна лечащему врачу, который занимается лечением своего пациента. То есть он может делать всё, что захочет, гибко настраивать систему под свои желания и нужды. Технологии VR хорошо подходит для пациентов, которые трудно поддаются традиционным методам лечения фобии, неспособных хорошо фантазировать и воображать, визуализировать.

В литературе есть убедительные доказательства терапевтического эффекта виртуальной реальности: снижение тревожности, стресса и страха перед операцией. Таким образом, технологии виртуальной реальности перспективны в области психологической подготовки пациентов к хирургическим вмешательствам в челюстно-лицевой области и представляют особый интерес для челюстно-лицевых хирургов, стоматологов и психологов.

\section{Описание концепции исследования}

Концепция данного исследования состоит в том, чтобы оценить эффективность, с которой технологии виртуальной реальности могут помочь пациентам стоматолога справиться с их страхами и стрессом, а также упростить работу самих врачей-стоматологов. Данная научноисследовательская работа является междисциплинарным проектом, выполняемым в сотрудничестве с коллегами из Первого Санкт-Петербургского государственного медицинского университета им. акад. И. П. Павлова, отделения челюстно-лицевой и пластической хирургии. Имеется реальный запрос - потребность коллег в повышении эффективности своей работы с помощь VR технологий с их последующим внедрением в практику.

После рассмотрения аналогов, обдумывания и обсуждения проекта, было решено создать виртуальную аутентичную локацию реального операционного стоматологического кабинета. Сотрудники университета использовали созданное VR приложение для подготовки своих пациентов к проведению стоматологических операций непосредственно перед их началом для облегчения их психологического состояния.

Поместив человека, страдающего дентофобией в виртуальное пространство, имитирующее стоматологический операционный кабинет, сотрудники клиники ставили цель помочь пациенту привыкнуть к окружающей обстановке, к атмосфере самой операционной в полной безопасности и покое, чтобы он, освоившись в своей фобической среде, успокоился, расслабился и перестал испытывать страх.

Если помочь пациенту изменить его поведение в ситуации, которую он считает опасной, с необоснованного и настороженного, на спокойное и адекватное, то можно и поменять его отношение к происходящему. Эмоциональные переживания человека основываются на том, как он относится к тому или иному событию.

Таким образом, созданное VR приложение способно, вкупе с работой психолога, помочь пациенту избавиться от фобии и уже спокойно посещать стоматолога. Именно оценка эффективности такого подхода и легла в основу данной научно-исследовательской работы.

\section{Обзор аналогов}

$\mathrm{B}$ настоящее время создано приличное количество медицинских VR приложений различного функционала и уровня сложности.

Культура и технологии, Том 6, № 2 
VR Flight - это система, созданная американским Медицинским центром виртуальной реальности, уже более 22 лет применяющим VR технологии в сочетании с физиологическим мониторингом и обратной связью для лечения пациентов с тревожными расстройствами, обучения как военных, так и гражданского населения, расширения различных образовательных программ (рис. 1).

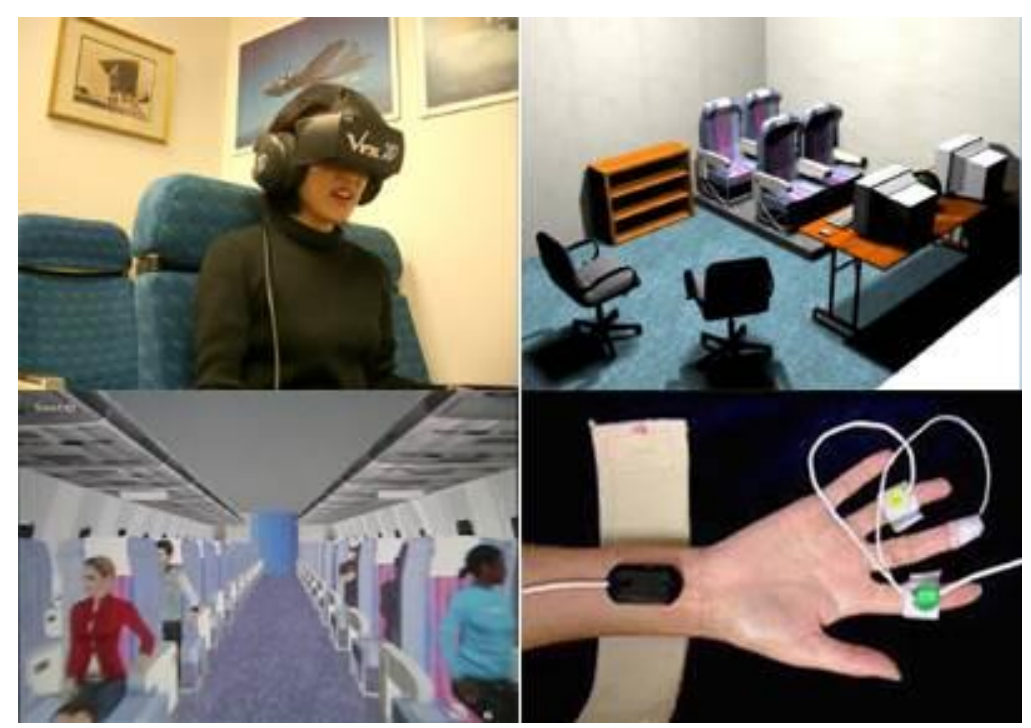

Рис. 1. Система VR Flight

VR Flight применяется для лечения боязни высоты (акрофобии) и полётов (аэрофобии). Пользователь сидит на реальном кресле коммерческого самолёта, со встроенным линейным двигателем и сабвуфером, что позволяет ему полностью погрузиться в атмосферу полёта на самолёте, почувствовать виртуальный взлёт, турбулентность или грозу, или же простые лёгкие вибрации от полёта. С включением объёмного звука, VR Flight симулятор может управлять визуальными, звуковыми и интерактивными аспектами опыта полёта в безопасных условиях клиники. Пользователь слышит голос капитана через громкоговоритель в самолёте, может смотреть в окно и видеть проходящие мимо облака, крыло самолёта, горизонт.

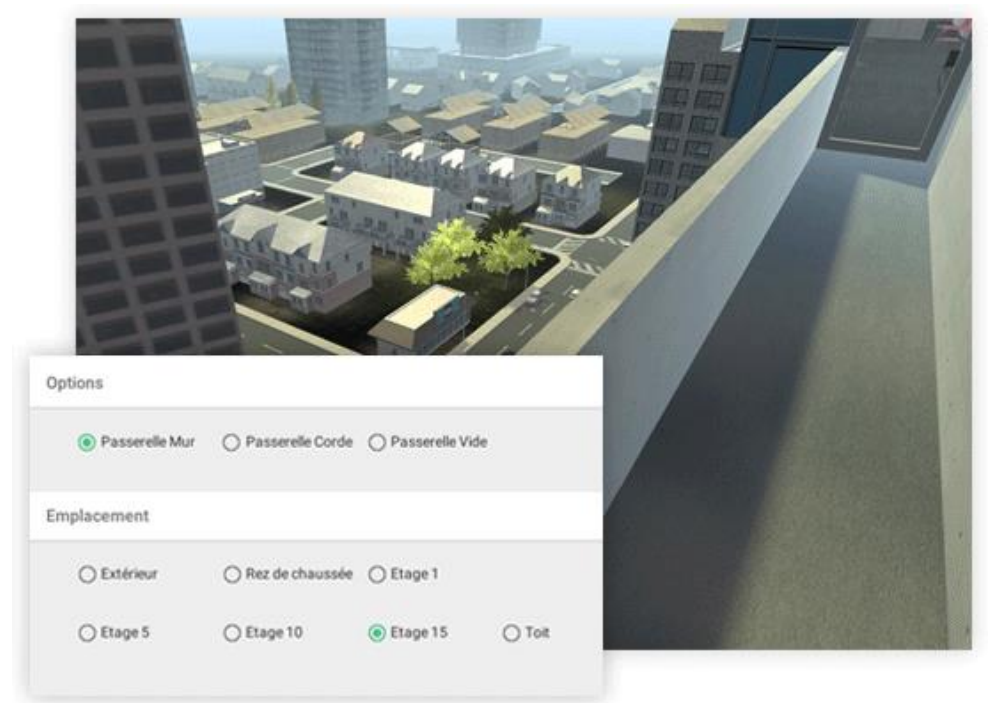

Рис. 2. Пример практического применения C2Phobia для лечения головокружения, боязни высоты

Весь процесс состоит в среднем из 8-12 сессий, проводит пациента через постепенно усиливающиеся симуляции различных ситуаций, использую гарнитуры виртуальной реальности и физиологический мониторинг в реальном времени и настраивается для каждого клиента

International Culture \& Technology Studies, Vol. 6, No. 2 
индивидуально. Приложение C2Phobia (рис. 2), созданное канадской компанией C2Care, состоит из более чем семидесяти виртуальных пространств для обеспечения лечения различных, самых распространённых фобий и тревожных расстройств, позволяющих постепенно и безопасно вводить пациентов в беспокоящие их условия окружающей среды.

Достоинства данной системы в её доступности и конфиденциальности, пациентам не нужно никуда ходить, систему можно использовать в домашних условиях. Более того, пользователи имеют возможность раз за разом повторять любые доступные сцены. Технология проста в применении и изучении.

Система может широко настраиваться для постепенного продвижения в лечении фобий. В зависимости от степени беспокойства и страха пациента врач может изменять условия окружающей среды, например, при боязни высоты можно менять высоту сцены (от первого до 15 этажа, или же до крыши небоскрёба).

\section{Разработка приложения виртуальной реальности}

Так как пациенты испытывают страх перед стоматологическими процедурами, было решено готовить пациентов к операции психологически посредством VR технологий. Гарнитура виртуальной реальности должна быть мобильной и простой в использовании, чтобы её могли применять сотрудники клиники без посторонней помощи и постоянной настройки оборудования. Нужно достичь максимального погружения пациента в атмосферу кабинета, подготовленного к проведению стоматологической операции. В сцене должны присутствовать все самые необходимые предметы, важные для передачи ощущений пациента через виртуальное пространство.

B процессе работы было создано VR приложение для гарнитуры Samsung Gear VR на движке Unity, с виртуальной моделью, имитирующей реальную операционную, в которой будет находиться пациент во время операции (рис. 3).

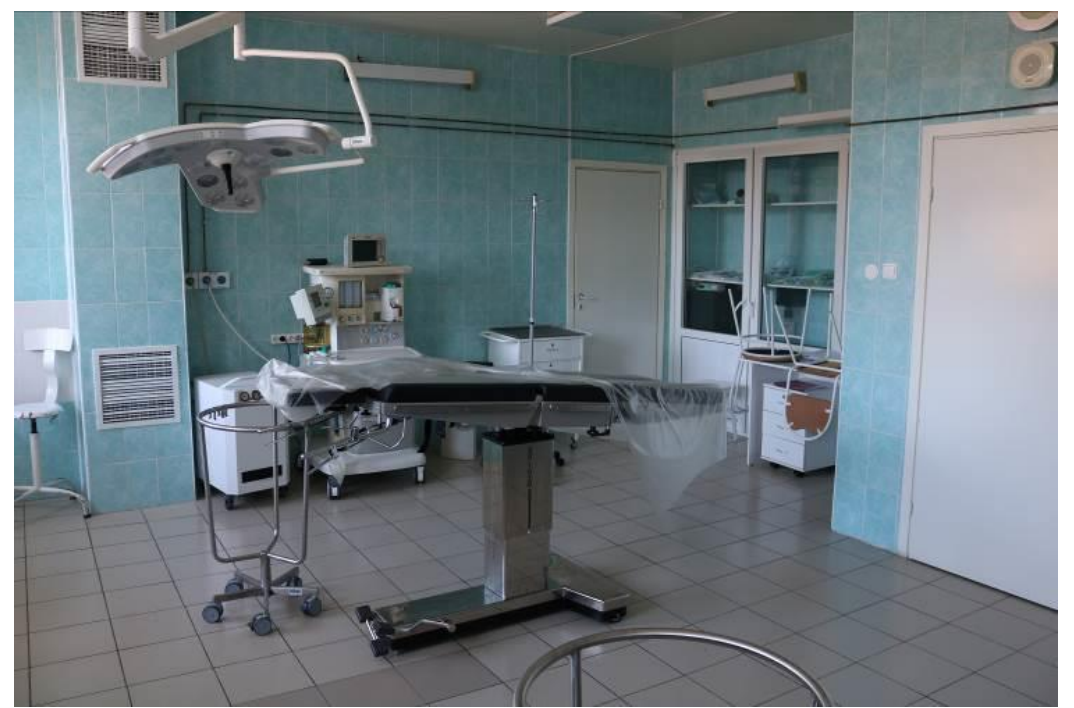

Рис. 3. Стоматологический кабинет-операционная клиники челюстно-лицевой и пластической хирургии

Предполагалось, что пациент будет находиться в лежачем положении, не будет совершать никаких движений кроме поворотов головы, поэтому часть моделей можно было упростить и делать низкополигональными, накладывая на них фототекстуры, для реалистичной имитации поверхностей и деталей объектов без больших затрат ресурсов системы, что очень важно, так как Samsung Gear VR - мобильная гарнитура с определенными ограничениями. Поэтому, при подготовке к реализации проекта сначала были выполнены все измерения реального стоматологического кабинета, сделаны необходимые фотоизображения, по которым строилась сцена, а также фотографии для создания фототекстур. После этого были созданы все 3D модели и собрана единая сцена (рис. $4,5,6)$. 
Эффективность использования технологий виртуальной реальности в методиках лечения дентофобии для облегчения психофизиологического состояния пациентов стоматолога

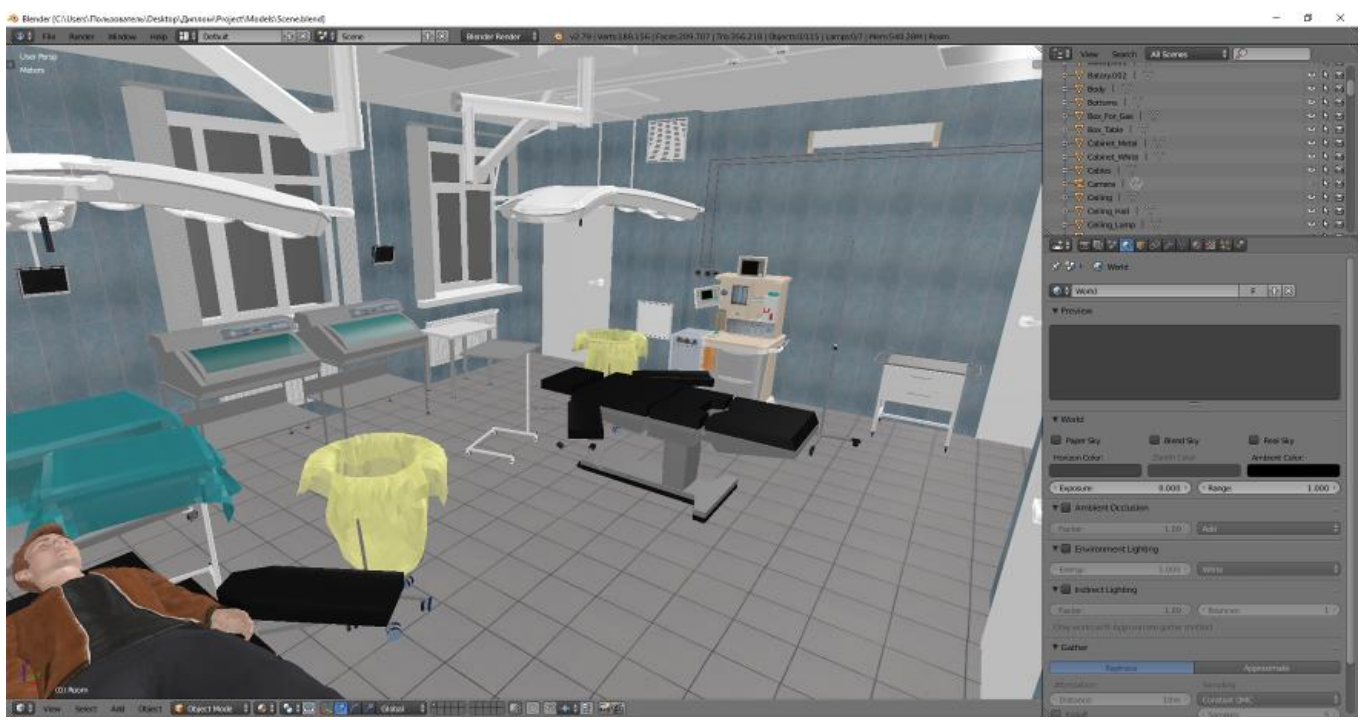

Рис. 4. Процесс работы над приложением

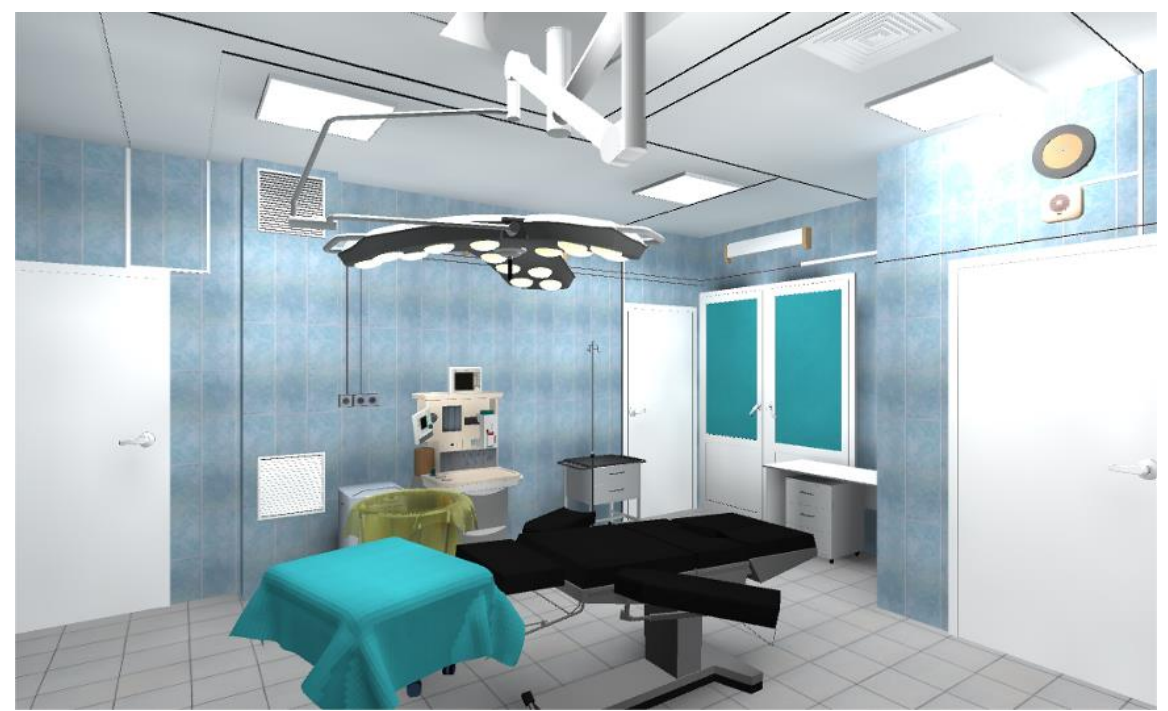

Рис. 5. Скриншот готовой виртуальной локации стоматологического операционного кабинета

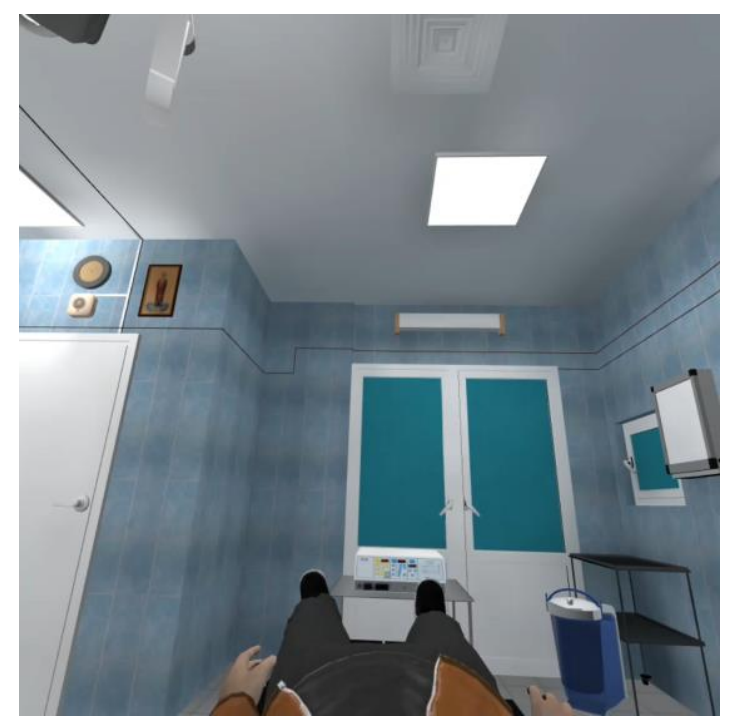

Pис. 6. Скриншот работы приложения, запущенного на гарнитуре виртуальной реальности Samsung Gear VR

International Culture \& Technology Studies, Vol. 6, No. 2 
В процессе параллельной работы с коллегами-медиками, было решено провести технический опрос удобства использования приложения через гарнитуру виртуальной реальности Samsung Gear VR. Одной из основных задач был комфорт респондентов, их ощущения и впечатления от устройства и приложения, то, что они чувствовали во время погружения в виртуальную реальность.

\section{Проведение тестирования}

В рамках работы над данным проектом было проведено тематическое тестирование различных пользователей на предмет их восприятия виртуальной реальности (рис. 7).

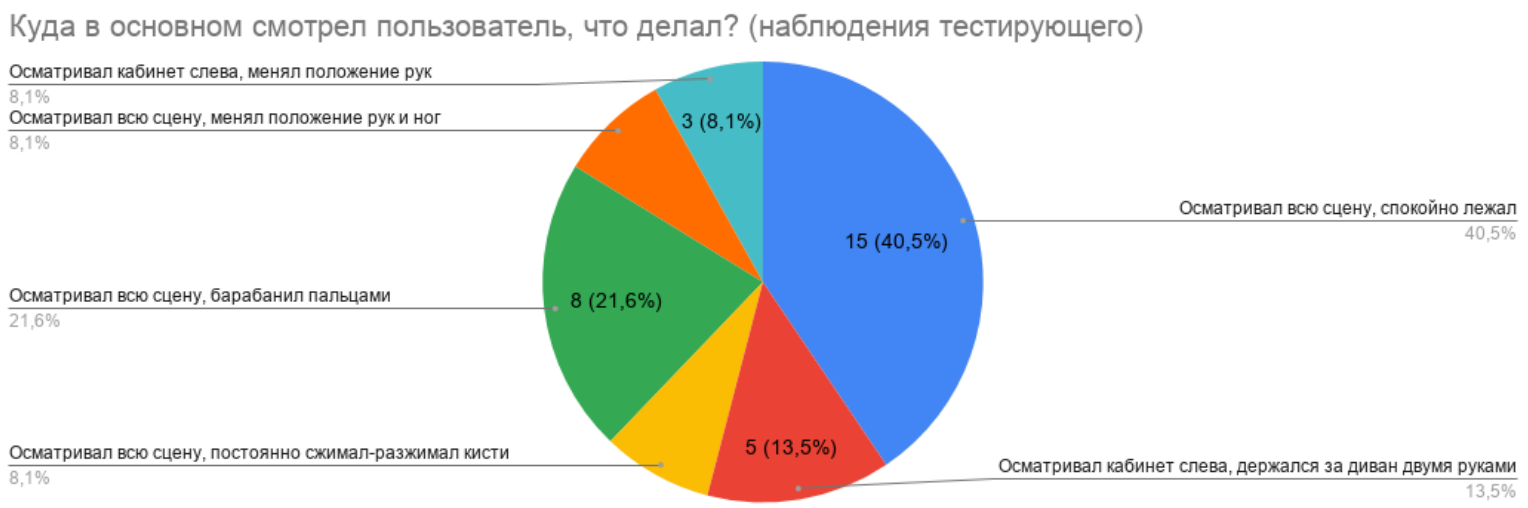

Рис. 7. График результата опроса-тестирования

Большинство опрошенных составляли люди мужского пола, испытывающие дискомфорт во время посещения стоматолога. Общее качество устройства $62 \%$ респондентов оценили высоко, $35 \%$ оценили, как хорошее. Качеством приложения респонденты остались довольны. Основные предложения и жалобы по работе приложения были связаны с графической составляющей просили добавить деталей и улучшить уровень графики, что, к сожалению, не позволяют мощности выбранной мобильной гарнитуры виртуальной реальности Samsung Gear VR.

Больше половины респондентов $(56,8 \%)$ остались довольны работой приложения. Само устройство не причиняло дискомфорта $70 \%$ опрошенным. Остальные жаловались на материалы устройства, дискомфорт на коже лица, потливость. Приложение особого дискомфорта не причиняло, 13,5\% опрошенных отметили недостаточную плавность изображения. Для большей достоверности и ощущения полного погружения в виртуальное пространство было предложено добавить звук подготовки к стоматологическим процедурам $(8,1 \%)$, улучшить графику $(13,5 \%)$ и добавить возможность двигаться во время сеанса $(13,5 \%)$.

Более половины респондентов (64,8\%) испытывали некоторую степень дискомфорта от пребывания в виртуальном пространстве, имитирующем кабинет стоматолога, 59,5\% респондентов во время сеанса вели себя беспокойно.

В результате опроса были сделаны выводы о том, что действительно получилось вызвать у пользователей ощущения необходимые для проведения основного эксперимента в контексте решения поставленных задач и проведения основного эксперимента.

\section{Выводы}

Итоговый проект виртуального пространства операционного стоматологического кабинета клиники челюстно-лицевой и пластической хирургии при Первом Санкт-Петербургском медицинском университете им. акад. И. П. Павлова для мобильной гарнитуры Samsung Gear VR будет применяться сотрудниками клиники в своей практике с пациентами, страдающими дентофобией, чтобы помочь им преодолеть негативные эмоции (рис. 8). 


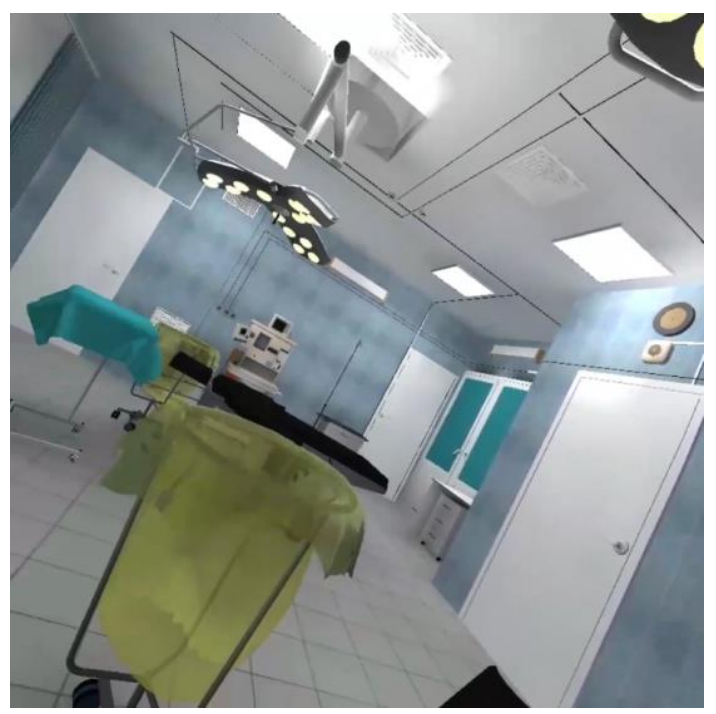

Рис. 8. В процессе работы приложения с экрана VR гарнитуры (видео, доступ по URL: https://youtu.be/9pZK-pyrtGE)

Выполненная работа обладает социальной значимостью, поскольку приложение поможет людям, страдающим дентофобией, избавиться от своих страхов, а сотрудникам клиники улучшить и упростить свою стоматологическую практику. Следовательно, созданное VR приложение способно показать, как профессионалам, так и простым пациентам преимущества и потенциал технологий в общем, и VR технологий в частности, что, в конечном итоге, будет способствовать популяризации технологий виртуальной реальности.

Дальнейшее развитие созданного проекта может заключаться в улучшении процесса погружения пользователя в виртуальное пространство, сделав его опыт более правдоподобным и достоверным.

\title{
Литература
}

[1] Milosevic I., McCabe R.E. Phobias: The Psychology of irrational Fear / Milosevic I., McCabe R.E. Greenwood, 2015. - 444 c.

[2] Beck A., Emery G., Greenberg R. Anxiety Disorders and Phobias: A Cognitive Perspective / Beck A., Emery G., Greenberg R. - BasicBooks, 2005. - 384 c.

[3] Bourne E.J. The Anxiety and Phobia Workbook / Bourne E.J. - New Harbinger Publication, 2011. - 488 c.

[4] Burdea G.C, Coiffet P. Virtual Reality Technology / Burdea G.C, Coiffet P. - Wiley-IEEE Press, 2003. - 464 c.

[5] Sherman W.R, Craig A.B. Understanding Virtual Reality: Interface, Application, and Design / Sherman W.R, Craig A.B. - Morgan Kaufmann, 2002. - 938 c.

[6] Papagiannis H. Augmented Human: How Technology Is Shaping the New Reality / Papagiannis H. - O'Reilly Media, 2017. - 200 c.

[7] Дентофобия: преодолеваем страх перед стоматологом // Фобия Инфо [Электронный ресурс]. - 2014. Режим доступа: http://fobiya.info/strah-situatsij/dentofobiya. - Дата доступа: 21.12.2020).

\section{Effectiveness of Virtual Reality Technologies Usage in Dentophobia Treatment Methods to Relief the Psychophysiological State of Dentist Patients}

\author{
N.A. Tulikov, A.A. Smolin \\ ITMO University, Russia
}

\begin{abstract}
Virtual reality is a current trend in the field of modern computer technology, which is widely used in many areas, especially in medicine. The aim of this scientific work is to test and evaluate the effectiveness of usage virtual reality technologies in methods of treating dentophobia by immersing a patient suffering from dentophobia into a virtual environment that imitates the operating
\end{abstract}

International Culture \& Technology Studies, Vol. 6, No. 2 
room of a dentist. Since patients are afraid of dental procedures, it was decided to prepare patients for surgery psychologically, using VR technologies. In the process of work, a virtual reality application was developed for the Samsung Gear VR headset, methods of using virtual reality and methods of interacting with it, psychological therapeutic theories were studied, software analysis for developing an application was carried out, and a hypothesis was formulated. A technical survey was conducted on the operation of the application, an experiment is planned.

Keywords: Virtual reality, dentophobia, app development, therapy, medicine, VR, headset, phobia, dentist, preoperative preparation, patients, fear, 3D, interdisciplinary research.

\section{References}

[1] Milosevic, I., McCabe, R.E. (2015) Phobias: The Psychology of irrational Fear. Greenwood. 444 p.

[2] Beck A., Emery G., Greenberg R. (2005) Anxiety Disorders and Phobias: A Cognitive Perspective. BasicBooks. 384 p.

[3] Bourne E.J. (2011) The Anxiety and Phobia Workbook. New Harbinger Publication. 488 p.

[4] Burdea G.C, Coiffet P. (2003) Virtual Reality Technology. Wiley-IEEE Press. 464 p.

[5] Sherman W.R, Craig A.B. (2002) Understanding Virtual Reality: Interface, Application, and Design. Morgan Kaufmann. 938 p.

[6] Papagiannis H. (2017) Augmented Human: How Technology Is Shaping the New Reality. O'Reilly Media. 200 p.

[7] Dentofobija: preodolevaem strah pered stomatologom. Fobija Info: Available at: http://fobiya.info/strahsituatsij/dentofobiya (Access date: 21.12.2020). 